\title{
Simultaneous identification of 36 mutations in KRAS codons 61and 146, BRAF, NRAS, and PIK3CA in a single reaction by multiplex assay kit
}

Hideaki Bando ${ }^{1}$, Takayuki Yoshino ${ }^{1 *}$, Eiji Shinozaki ${ }^{2}$, Tomohiro Nishina ${ }^{3}$, Kentaro Yamazaki ${ }^{4}$, Kensei Yamaguchi ${ }^{5}$, Satoshi Yuki ${ }^{6}$, Shinya Kajiura ${ }^{7}$, Satoshi Fujiii ${ }^{8}$, Takeharu Yamanaka ${ }^{9}$, Katsuya Tsuchihara ${ }^{9}$ and Atsushi Ohtsu ${ }^{1,9}$

\begin{abstract}
Background: Retrospective analyses in the West suggest that mutations in KRAS codons 61 and 146, BRAF, NRAS, and PIK3CA are negative predictive factors for cetuximab treatment in colorectal cancer patients. We developed a novel multiplex kit detecting 36 mutations in KRAS codons 61 and 146, BRAF, NRAS, and PIK3CA using Luminex (XMAP) assay in a single reaction.
\end{abstract}

Methods: Tumor samples and clinical data from Asian colorectal cancer patients treated with cetuximab were collected. We investigated KRAS, BRAF, NRAS, and PIK3CA mutations using both the multiplex kit and direct sequencing methods, and evaluated the concordance between the 2 methods. Objective response, progression-free survival (PFS), and overall survival (OS) were also evaluated according to mutational status.

Results: In total, 82 of 83 samples (78 surgically resected specimens and 5 biopsy specimens) were analyzed using both methods. All multiplex assays were performed using $50 \mathrm{ng}$ of template DNA. The concordance rate between the methods was 100\%. Overall, 49 (59.8\%) patients had all wild-type tumors, 21 (25.6\%) had tumors harboring KRAS codon 12 or 13 mutations, and 12 (14.6\%) had tumors harboring KRAS codon 61, KRAS codon 146, BRAF, NRAS, or PIK3CA mutations. The response rates in these patient groups were $38.8 \%, 4.8 \%$, and $0 \%$, respectively. Median PFS in these groups was 6.1 months (95\% confidence interval (Cl): 3.1-9.2), 2.7 months (1.2-4.2), and 1.6 months (1.5-1.7); median OS was 13.8 months (9.2-18.4), 8.2 months (5.7-10.7), and 6.3 months (1.3-11.3), respectively. Statistically significant differences in both PFS and OS were found between patients with all wild-type tumors and those with KRAS codon 61, KRAS codon 146, BRAF, NRAS, or PIK3CA mutations (PFS: $95 \% \mathrm{Cl}, 0.11-0.44 ; P<0.0001$; OS: $95 \% \mathrm{Cl}, 0.15-0.61 ; P<0.0001$ ).

Conclusions: Our newly developed multiplex kit is practical and feasible for investigation of a range of sample types. Moreover, mutations in KRAS codon 61, KRAS codon 146, BRAF, NRAS, or PIK3CA detected in Asian patients were not predictive of clinical benefits from cetuximab treatment, similar to the result obtained in European studies.

Keywords: Luminex assay, KRAS, BRAF, NRAS, PIK3CA, Epidermal growth factor

\section{Background}

The clinical significance of KRAS codon 12 and $13 \mathrm{mu}-$ tation tests in the selection of patients with colorectal cancer who might benefit from anti-epidermal growth factor receptor (EGFR) antibodies is well established, and regulatory authorities in Europe, the United States,

\footnotetext{
* Correspondence: tyoshino@east.ncc.go.jp

'Department of Gastroenterology and Gastrointestinal Oncology, National Cancer Center Hospital East, 6-5-1 Kashiwanoha, Kashiwa, Chiba 277-8577, Japan

Full list of author information is available at the end of the article
}

and Japan have recommended compulsory KRAS mutation testing before treatment [1-6]. Although conventional KRAS tests are useful to decrease treatment to nonbeneficiary populations, the efficacy of determining beneficiary populations requires improvement. The response rate to anti-EGFR antibody monotherapy among pretreated patients with tumors harboring KRAS codons 12 and 13 wild-type is $13 \%-17 \%[1,2]$, and that of combination anti-EGFR antibody and cytotoxic agent therapy is $11 \%-35 \%[5,7]$. One explanation for such relatively low efficacy is that molecular alterations other than KRAS codon

\section{Biomed Central}

(c) 2013 Bando et al.; licensee BioMed Central Ltd. This is an Open Access article distributed under the terms of the Creative Commons Attribution License (http://creativecommons.org/licenses/by/2.0), which permits unrestricted use, distribution, and reproduction in any medium, provided the original work is properly cited. 
12 and 13 mutations might confer resistance to anti-EGFR antibody therapies. Recent retrospective studies have revealed that mutations in KRAS codons 61 and 146, BRAF, $N R A S$, and PIK3CA are also related to resistance to antiEGFR antibodies [8-13].

Several issues should also be considered to establish the clinical utility of expanded genome biomarker tests for anti-EGFR antibodies. First, information about the relation between mutation status and efficacy of treatment, especially among Asian populations, is still limited. Second, efficacious quality-controlled in vitro diagnostic kits and systems suitable for multiple genome biomarker detection are needed.

In Japan, a KRAS mutation assay kit based on the ARMS-scorpion method that detects seven frequently observed mutations in KRAS codons 12 and 13 (TheraScreen ${ }^{\circledR}$ K-RAS Mutation Kit; QIAGEN) was first approved for in vitro diagnostic use, and a kit using Luminex (xMAP) assay (MEBGEN KRAS Mutation Detection Kit, MBL) followed $[14,15]$. We recently developed another Luminexbased research-use kit, GENOSEARCH Mu-PACK, which simultaneously detects 36 mutations in KRAS codons 61 and 146, BRAF, NRAS, and PIK3CA. In addition to the hitherto approved KRAS codon 12 and 13 mutation kit, the multiplex kit identifies mutations by a single tube reaction using $50 \mathrm{ng}$ of template DNA from formalin-fixed paraffin-embedded (FFPE) specimens.

In this study, we examined the feasibility and robustness of this multiplex kit using routine clinical samples collected from multiple hospitals. Meanwhile, we collected precise clinical data for these cases and retrospectively analyzed the relation of the mutation profiles of expanded markers to clinical outcomes following cetuximab therapy.

\section{Methods \\ Patients}

We screened and selected clinical and pathological data from consecutive patients who were administered either cetuximab monotherapy or cetuximab plus irinotecan between July 2008 and April 2010.

Patients who met all of the following inclusion criteria were retrospectively included in the analyses: (1) age $\geq 20$ years; (2) histologically confirmed adenocarcinoma of the colon or rectum; (3) presence of unresectable metastatic disease; (4) baseline computed tomography (CT) performed within 28 days of initial cetuximab administration; (5) initial CT evaluation performed within 3 months of initial cetuximab administration; (6) previously documented as refractory or intolerant to fluoropyrimidines, oxaliplatin, and irinotecan; (7) Eastern Cooperative Oncology Group performance status score $\leq 2$; and (8) adequate hematological, hepatic, and renal functions.

In the monotherapy regimen, cetuximab was administered at an initial dose of $400 \mathrm{mg} / \mathrm{m}^{2}$ followed by weekly infusions of $250 \mathrm{mg} / \mathrm{m}^{2}$. In the cetuximab plus irinotecan regimen, cetuximab was administered at the same dose as for monotherapy and followed by biweekly infusions of $150 \mathrm{mg} / \mathrm{m}^{2}$ irinotecan, as per the manufacturer's instructions for irinotecan in Japan.

The study was conducted with the approval of the $\mathrm{Na}$ tional Cancer Center Institutional Review Board, Cancer Institute Hospital of Japanese Foundation for Cancer Research Review Board, National Hospital Organization Shikoku Cancer Center Review Board, Shizuoka Cancer Center Review Board, Saitama Cancer Center Review Board, Hokkaido University Review Board, and the Ethics Committee of the University of Toyama. Written informed consent was obtained from as much patients who were alive as possible. For the deceased patients and their relatives, we also disclosed the study design at the website of National Cancer Center and gave them chances to express their wills in accordance with Epidemiological Study Guideline of Ministry of Health, Labour and Welfare in Japan.

\section{Tissue samples and DNA extraction}

Genomic DNA was obtained from primary and metastatic colorectal cancer tissues of all patients treated with cetuximab. Tissue samples harvested by biopsy or surgical resection at the participating hospitals were collected and sent to the research institution (MBL, Japan). A 2- $\mu \mathrm{m}$ hematoxylin-eosin (HE) slide and a 10- $\mu \mathrm{m}$ unstained slide were obtained from the FFPE tissue blocks; the latter was subsequently sliced into 3-10 sections. Pathological diagnoses were confirmed by a pathologist (Satoshi Fujii), with reference to the $4^{\text {th }}$ edition of the WHO classification. The tumor area, determined by examining HE slides, was macroscopically dissected. Genomic DNA was isolated as described previously [16].

\section{Luminex (xMAP) tests}

A total of 36 mutations of $K R A S$ codon 61 (Q61K, Q61E, Q61L, Q61P, Q61R, Q61H), KRAS codon 146 (A146T, A146S, A146P, A146E, A146V, A146G), BRAF codon 600 (V600E), NRAS codon 12 (G12S, G12C, G12R, G12D, G12V, G12A), codon 13 (G13S, G13C, G13R, G13D, G13V, G13A), codon 61 (Q61K, Q61E, Q61L, Q61P, Q61R, Q61H), PIK3CA exon 9 codon 542 (E542K), codon 545 (E545K), codon 546 (E546K), and exon 20 codon 1047 (H1047R, H1047L) were analyzed using Luminex (xMAP) technology (GENOSEARCH Mu-PACK, MBL, Japan).

First, 50 ng of template DNA collected from FFPE tissue samples was amplified by polymerase chain reaction (PCR) using a biotin-labeled primer. Thereafter, the PCR products and fluorescent Luminex beads (oligonucleotide probes complementary to wild and mutant genes were bound to the beads) were hybridized and labeled with streptavidin-phycoerythrin. Subsequently, the products 
were processed by Luminex assay and the collected data analyzed using UniMAG software (MBL, Japan). The procedure time was approximately $4.5 \mathrm{~h}$.

We also used the Luminex assay kit (MEBGEN KRAS Mutation Detection Kit, MBL, Japan) currently approved for clinical use by the Ministry of Health, Labour and Welfare of Japan [16] to detect KRAS codon 12 and 13 mutations.

\section{Direct sequencing methods}

In addition, to confirm the mutations detected by the Luminex assays, the same mutations of KRAS codons 61 and 146, BRAF, NRAS, and PIK3CA were analyzed by direct sequencing. A total of $700 \mathrm{ng}$ of template DNA was used for these PCR reactions and the PCR products were directly sequenced with the same primers used for PCR. A BigDye Terminator v3.1 Cycle Sequencing Kit and an ABI PRISM 3730xl DNA Analyzer (Life Technologies) were used. Analyses of DNA sequences were performed using Sequencher (GeneCodes).

\section{Statistical analysis}

Response rates (RRs) and disease control rates (DCRs) (including complete or partial response and stable disease) were evaluated as per the Response Evaluation Criteria in Solid Tumors (RECIST) (version 1.0). Progressionfree survival (PFS) was defined as the time from initial administration of a cetuximab-containing regimen to either the first objective evidence of disease progression or death from any cause. Overall survival (OS) was defined as the time from initial administration of a cetuximabcontaining regimen to death from any cause. RRs, DCRs, PFS, and OS of all patients were re-evaluated by the principal investigators at each institution. The relative dose intensity was defined as the ratio of the actual dose administered to the planned dose.

Fisher's exact test and the Kruskal-Wallis test were used to compare patient characteristics, relative dose intensity, and treatment response. PFS and OS data were plotted as Kaplan-Meier curves, and differences among the groups according to KRAS, BRAF, NRAS, and PIK3CA gene status were compared using the log-rank test and hazard ratio calculated from a Cox regression model with a single covariate. All analyses were performed by a biostatistician (Takeharu Yamanaka), using IBM SPSS ${ }^{\circ}$ Statistics 21 package software (SPSS Inc., Tokyo, Japan).

\section{Results}

Concordance between Luminex and direct sequencing

From September 2008 to April 2010, 376 patients were treated with a cetuximab-containing regimen at seven institutions. Of these, 83 patients met the inclusion criteria and specimens were collected from them for analysis (232 patients did not meet the inclusion criteria and 61 specimens could not be collected). We collected 78 surgically resected specimens and 5 biopsy specimens, from which the median amount of template DNA collected was 25,114 ng (range: 274084,738) and $1691 \mathrm{ng}$ (range:1469-2668), respectively (Table 1).

One patient's gene status could not be detected by either Luminex or direct sequencing because DNA harvested from the resected metastatic liver specimens could not be amplified by PCR. In the remaining 82 patients, the concordance rate for mutations between the 2 methods was $100 \%$ (Table 2).

Among the 82 specimens, 3 KRAS codon 61 mutations (3.6\%), 2 KRAS codon 146 mutations (2.4\%), 4 BRAF mutations (4.9\%), 2 NRAS mutations (2.4\%), and 4 PIK3CA mutations (4.9\%) (1 in exon 9 and 3 in exon 20) were detected using both the expanded kit and direct sequencing. Moreover, we identified 15 KRAS codon 12 mutations (18.3\%) and 6 KRAS codon 13 mutations (7.3\%); in total, 21 samples $(25.6 \%)$ with $K R A S$ codon 12 or 13 mutations were detected by using the KRAS Luminex assay kit. All mutations except for PIK3CA were mutually exclusive (Table 2, Figure 1).

\section{Patient characteristics}

Clinical data were collected from 83 patients. We used data from 82 patients whose genomic DNA could be successfully examined using both the expanded kit and direct sequencing. Six of the 82 patients were treated with cetuximab monotherapy, while the remaining 76 were treated with a regimen of cetuximab plus irinotecan.

Of these 82 patients, 49 had tumors with no mutation (all wild type), 21 had tumors with mutation of either KRAS codon 12 or 13 , and 12 had tumors with mutation of either KRAS codon 61, KRAS codon 146, BRAF, $N R A S$, or PIK3CA. No significant difference was observed in the characteristics of these three groups except for the ratio of refractoriness to intolerance of prior oxaliplatin (Table 3).

Table 1 Template DNA harvested from FFPE specimens

\begin{tabular}{llll}
\hline & Surgically resected & Biopsy & \multicolumn{1}{c}{ Total } \\
\hline Number of specimens & 78 & 5 & 83 \\
Total amount of template DNA (ng) [median (range)] & $25,114(2,740-84,738)$ & $1,691(1,469-2,668)$ & $22,591(1,469-84,738)$ \\
Amount of template DNA per slice (ng) [median (range)] & $8,371(914-28,246)$ & $370(154-889)$ & $7,530(154-28,246)$ \\
\hline
\end{tabular}


Table 2 Concordance between Luminex and direct sequencing

\begin{tabular}{|c|c|c|c|c|}
\hline Gene & $\begin{array}{l}\text { Direct } \\
\text { sequencing } \\
\text { (DS) }\end{array}$ & Luminex & $\begin{array}{l}\text { Concordance } \\
\text { rate }\end{array}$ & $\begin{array}{l}\text { Mutation } \\
\text { rate }\end{array}$ \\
\hline KRAS codon 61 & 3 & 3 & $100 \%$ & $3.6 \%$ \\
\hline Q61K & 0 & 0 & $100 \%$ & $0 \%$ \\
\hline Q61E & 0 & 0 & $100 \%$ & $0 \%$ \\
\hline Q61L & 0 & 0 & $100 \%$ & $0 \%$ \\
\hline Q61P & 0 & 0 & $100 \%$ & $0 \%$ \\
\hline Q61R & 0 & 0 & $100 \%$ & $0 \%$ \\
\hline Q61H & 3 & 3 & $100 \%$ & $3.6 \%$ \\
\hline KRAS codon 146 & 2 & 2 & $100 \%$ & $2.4 \%$ \\
\hline A146T & 2 & 2 & $100 \%$ & $2.4 \%$ \\
\hline A146S & 0 & 0 & $100 \%$ & $0 \%$ \\
\hline $\mathrm{A} 146 \mathrm{P}$ & 0 & 0 & $100 \%$ & $0 \%$ \\
\hline A146E & 0 & 0 & $100 \%$ & $0 \%$ \\
\hline A146V & 0 & 0 & $100 \%$ & $0 \%$ \\
\hline A146G & 0 & 0 & $100 \%$ & $0 \%$ \\
\hline BRAF codon 600 & 4 & 4 & $100 \%$ & $4.9 \%$ \\
\hline V600E & 4 & 4 & $100 \%$ & $4.9 \%$ \\
\hline NRAS codon 12 & 2 & 2 & $100 \%$ & $2.4 \%$ \\
\hline G12S & 0 & 0 & $100 \%$ & $0 \%$ \\
\hline $\mathrm{G} 12 \mathrm{C}$ & 0 & 0 & $100 \%$ & $0 \%$ \\
\hline G12R & 0 & 0 & $100 \%$ & $0 \%$ \\
\hline G12D & 2 & 2 & $100 \%$ & $2.4 \%$ \\
\hline G12V & 0 & 0 & $100 \%$ & $0 \%$ \\
\hline G12A & 0 & 0 & $100 \%$ & $0 \%$ \\
\hline NRAS codon 13 & 0 & 0 & $100 \%$ & $0 \%$ \\
\hline G13S & 0 & 0 & $100 \%$ & $0 \%$ \\
\hline G13C & 0 & 0 & $100 \%$ & $0 \%$ \\
\hline G13R & 0 & 0 & $100 \%$ & $0 \%$ \\
\hline G13D & 0 & 0 & $100 \%$ & $0 \%$ \\
\hline G13V & 0 & 0 & $100 \%$ & $0 \%$ \\
\hline G13A & 0 & 0 & $100 \%$ & $0 \%$ \\
\hline NRAS codon 61 & 0 & 0 & $100 \%$ & $0 \%$ \\
\hline Q61K & 0 & 0 & $100 \%$ & $0 \%$ \\
\hline Q61E & 0 & 0 & $100 \%$ & $0 \%$ \\
\hline Q61L & 0 & 0 & $100 \%$ & $0 \%$ \\
\hline Q61P & 0 & 0 & $100 \%$ & $0 \%$ \\
\hline Q61R & 0 & 0 & $100 \%$ & $0 \%$ \\
\hline Q61H & 0 & 0 & $100 \%$ & $0 \%$ \\
\hline PIK3CA Exon 9 & 1 & 1 & $100 \%$ & $1.2 \%$ \\
\hline E542K & 1 & 1 & $100 \%$ & $1.2 \%$ \\
\hline E545K & 0 & 0 & $100 \%$ & $0 \%$ \\
\hline E546K & 0 & 0 & $100 \%$ & $0 \%$ \\
\hline
\end{tabular}

Table 2 Concordance between Luminex and direct sequencing (Continued)

\begin{tabular}{lllll}
\hline PIK3CA Exon 20 & $\mathbf{3}$ & $\mathbf{3}$ & $\mathbf{1 0 0 \%}$ & $\mathbf{3 . 7 \%}$ \\
H1047R & 1 & 1 & $100 \%$ & $1.2 \%$ \\
H1047L & 2 & 2 & $100 \%$ & $2.4 \%$ \\
\hline
\end{tabular}

\section{Response to treatment}

RRs of patients with all wild-type tumors $(N=49), K R A S$ codon 12 or 13 mutations $(N=21)$, and mutations of KRAS codon 61, KRAS codon 146, BRAF, NRAS, or PIK3CA $(N=12)$ were $38.8 \%, 4.8 \%$, and $0 \%$, respectively (Table 4). Partial response was observed in one patient with a KRAS codon G12C mutation. In addition, DCRs were $77.6 \%, 57.1 \%$, and $33.3 \%$, respectively, for these patient groups (Table 4). Differences for both RRs and DCRs between patients with all wild-type tumors and those with KRAS codon 61, KRAS codon 146, BRAF, NRAS, or PIK3CA mutations were statistically significant (Fisher's exact test, RRs: $P=0.006$, DCRs: $P=0.006$ ). On the other hand, there were no statistically significant differences between patients with KRAS codon 12 or 13 mutations and those with KRAS codon 61, KRAS codon 146, $B R A F$, NRAS, or PIK3CA mutations (Fisher's exact test, RRs: $P=0.636$, DCRs: $P=0.170$ ).

The relative dose intensity of cetuximab was significantly higher among patients with KRAS codon 61, KRAS codon 146, BRAF, NRAS, or PIK3CA mutations. However, the number of treatment cycles was significantly greater among patients with all wild-type tumors (Table 4).

RR for all patients included in the study was $24.4 \%$, whereas that for patients with KRAS codon 12 or 13 wildtype tumors was $31.1 \%$. Furthermore, RR for patients with all wild-type tumors was $38.8 \%$.

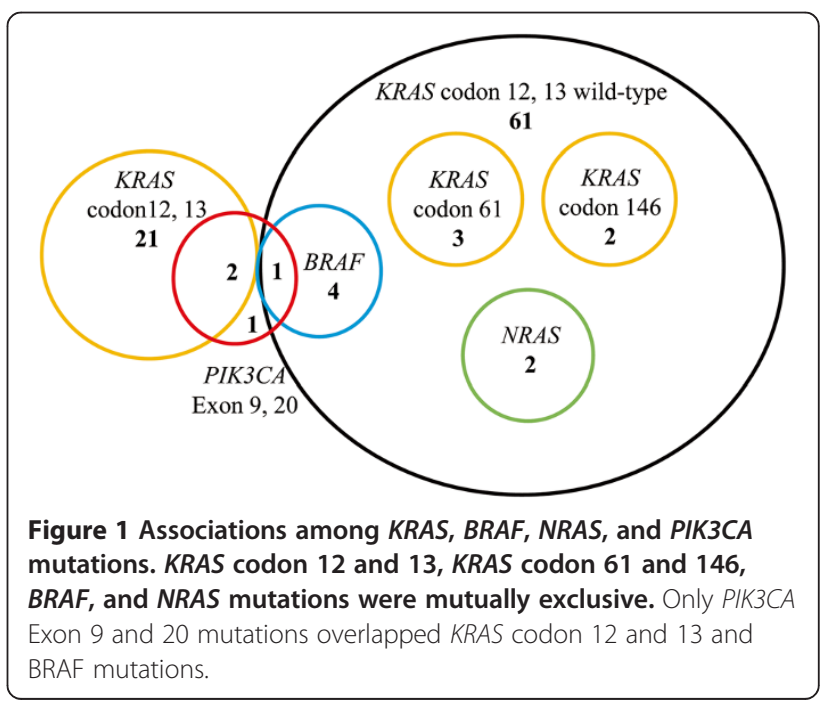


Table 3 Baseline patient characteristics

\begin{tabular}{|c|c|c|c|c|}
\hline & $\begin{array}{l}\text { All wild-type } \\
(N=49)\end{array}$ & $\begin{array}{l}\text { KRAS codon } 12, \\
13 \text { mutations } \\
(N=21)\end{array}$ & $\begin{array}{l}\text { KRAS codon } 61, \text { codon } 146, B R A F, N R A S \\
\text { or PIK3CA mutations (any other mutations) } \\
(N=12)\end{array}$ & \\
\hline \multicolumn{5}{|l|}{ Treatment } \\
\hline Cetuximab + irinotecan (\%) & $47(96)$ & $19(90)$ & $10(83)$ & \multirow[t]{2}{*}{$P=0.212$} \\
\hline Cetuximab monotherapy (\%) & $2(4)$ & $2(10)$ & $2(17)$ & \\
\hline \multicolumn{5}{|l|}{ Age } \\
\hline Median (range) & $61(29-78)$ & $65(51-80)$ & $65(43-76)$ & $P=0.605^{\ddagger}$ \\
\hline \multicolumn{5}{|l|}{ Gender } \\
\hline Male (\%) & $31(63)$ & $16(76)$ & $6(50)$ & \multirow[t]{2}{*}{$P=0.312$} \\
\hline Female (\%) & $18(37)$ & $5(24)$ & $6(50)$ & \\
\hline \multicolumn{5}{|l|}{ ECOG PS } \\
\hline $0(\%)$ & $34(69)$ & $13(62)$ & $5(42)$ & \multirow[t]{2}{*}{$P=0.185$} \\
\hline $1-2(\%)$ & $15(31)$ & $8(38)$ & $7(58)$ & \\
\hline \multicolumn{5}{|l|}{ Primary lesion } \\
\hline Colon (\%) & $28(57)$ & $15(71)$ & $9(75)$ & \multirow[t]{2}{*}{$P=0.416$} \\
\hline Rectum (\%) & $21(43)$ & $6(29)$ & $3(25)$ & \\
\hline \multicolumn{5}{|l|}{ Site of Metastasis } \\
\hline \multicolumn{5}{|l|}{ Liver } \\
\hline Yes (\%) & $33(67)$ & $13(62)$ & $8(67)$ & \multirow[t]{2}{*}{$P=0.945$} \\
\hline No (\%) & $16(33)$ & $8(38)$ & $3(33)$ & \\
\hline \multicolumn{5}{|l|}{ Lung } \\
\hline Yes (\%) & $34(69)$ & $15(71)$ & $9(75)$ & \multirow[t]{2}{*}{$P=1.000$} \\
\hline No (\%) & $15(31)$ & $6(29)$ & $3(25)$ & \\
\hline \multicolumn{5}{|l|}{ Lymph node } \\
\hline Yes (\%) & $26(53)$ & $7(33)$ & $9(75)$ & \multirow[t]{2}{*}{$P=0.068$} \\
\hline No (\%) & $23(47)$ & $14(67)$ & $3(25)$ & \\
\hline \multicolumn{5}{|l|}{ Peritoneum } \\
\hline Yes (\%) & $11(22)$ & $3(14)$ & $2(17)$ & \multirow[t]{2}{*}{$P=0.791$} \\
\hline No (\%) & $38(78)$ & $18(86)$ & $9(83)$ & \\
\hline \multicolumn{5}{|l|}{ No. of metastatic sites } \\
\hline $1(\%)$ & $9(18)$ & $9(42)$ & $3(25)$ & \multirow[t]{2}{*}{$P=0.106^{\dagger}$} \\
\hline$>2(\%)$ & $40(82)$ & $12(58)$ & $9(75)$ & \\
\hline \multicolumn{5}{|l|}{ Prior chemotherapy } \\
\hline \multicolumn{5}{|l|}{ Fluoropyrimidine } \\
\hline Refractory (\%) & $49(100)$ & $21(100)$ & $12(100)$ & \\
\hline Intolerant (\%) & $0(0)$ & $0(0)$ & $0(0)$ & \\
\hline \multicolumn{5}{|l|}{ Oxaliplatin } \\
\hline Refractory (\%) & $40(82)$ & $10(48)$ & $9(75)$ & \multirow[t]{2}{*}{$P=0.017^{\dagger}$} \\
\hline Intolerant (\%) & $9(18)$ & $11(52)$ & $3(25)$ & \\
\hline Irinotecan & & & & $P=1.000^{\dagger}$ \\
\hline Refractory (\%) & $48(98)$ & $21(100)$ & $12(100)$ & \\
\hline Intolerant (\%) & $1(2)$ & $0(0)$ & $0(0)$ & $P=0.669^{\dagger}$ \\
\hline
\end{tabular}


Table 3 Baseline patient characteristics (Continued)

\begin{tabular}{|c|c|c|c|c|}
\hline Before bevacizumab therapy & $25(51)$ & $9(43)$ & $7(58)$ & \\
\hline Yes (\%) & $24(49)$ & $12(57)$ & $5(42)$ & $P=0.236^{\dagger}$ \\
\hline No (\%) & 12 & 5 & 25 & \\
\hline \multicolumn{5}{|c|}{$\begin{array}{l}\text { Response rate for prior irinotecan-containing } \\
\text { therapies (\%) }\end{array}$} \\
\hline \multicolumn{5}{|l|}{ Pathological classification } \\
\hline G1, G2 (\%) & $42(86)$ & $20(95)$ & $11(92)$ & $P=0.481^{\dagger}$ \\
\hline G3, G4 (\%) & $7(14)$ & $1(5)$ & $1(8)$ & \\
\hline
\end{tabular}

ECOG PS Eastern Cooperative Oncology Group performance status.

${ }^{\dagger}$ : Fisher's exact test.

${ }^{\ddagger}$ : Kruskal-Wallis test.

\section{Survival}

The median PFS among patients with all wild-type tumors $(N=49), K R A S$ codon 12 or 13 mutations $(N=21)$, and KRAS codon 61, KRAS codon 146, BRAF, NRAS, or PIK3CA mutations $(N=12)$ was 6.1 months $(95 \%$ confidence interval (CI) 3.1-9.2), 2.7 months (1.2-4.2), and 1.6 months (1.5-1.7), respectively (Table 4, Figure 2A). Median OS was 13.8 months (9.2-18.4), 8.2 months (5.710.7 ), and 6.3 months (1.3-11.3), respectively (Table 4 , Figure 2B).

We observed statistically significant differences in both PFS and OS between patients with all wild-type tumors and those with KRAS codon 61, KRAS codon 146, BRAF,
NRAS, or PIK3CA mutations [PFS: hazard ratio (HR), 0.22; 95\% CI, 0.11-0.44; $P<0.0001$ ] (OS: HR, 0.30; 95\% CI, $0.15-0.61 ; P<0.0001$ ) (Figure $2 \mathrm{~A}$ and $2 \mathrm{~B}$ ). Differences in PFS and OS between patients with wild-type mutations and the 8 patients with KRAS codon 61, KRAS codon 146, NRAS, or PIK3CA mutations were statistically significant (PFS: $\mathrm{P}=0.001$, OS: $\mathrm{P}=0.001$ ), but this was not the case for the 4 patients with $B R A F$ mutations. The median PFS and OS for these 4 patients were 0.9 months and 11.4 months, respectively.

On the other hand, there were no statistically significant differences between patients with KRAS codon 12 or 13 mutations and those with KRAS codon 61, KRAS

Table 4 Efficacy in the test population determined on the basis of gene status

\begin{tabular}{|c|c|c|c|c|}
\hline & $\begin{array}{l}\text { All wild-type } \\
(N=49)\end{array}$ & $\begin{array}{l}\text { KRAS codon } 12,13 \\
\text { mutations }(N=21)\end{array}$ & $\begin{array}{l}\text { KRAS codon } 61 \text {, codon } 146, B R A F \text {, } \\
\text { NRAS or PIK3CA mutations } \\
\text { (any other mutations) }(N=12)\end{array}$ & \\
\hline Complete response & 1 & 0 & 0 & \\
\hline Partial response & 18 & 1 & 0 & \\
\hline Stable disease & 19 & 11 & 4 & \\
\hline Progressive disease & 11 & 9 & 8 & \\
\hline Total & 49 & 21 & 12 & \\
\hline Response rate (\%) & 38.8 & 4.8 & 0 & $\begin{array}{l}P=0.006^{*} \text { (All wild-type vs. } \\
\text { Any other mutations) }\end{array}$ \\
\hline Disease control rate (\%) & 77.6 & 57.1 & 33.3 & $\begin{array}{l}P=0.006^{*} \text { (All wild-type vs. } \\
\text { Any other mutations) }\end{array}$ \\
\hline $\begin{array}{l}\text { Progression-free survival } \\
\text { [Median }(95 \% \text { Cl) (months)] }\end{array}$ & $6.1(3.1,9.2)$ & $2.7(1.2,4.2)$ & $1.6(1.5,1.7)$ & $\begin{array}{l}P<0.0001^{* *} \text { (All wild-type vs. } \\
\text { Any other mutations) }\end{array}$ \\
\hline $\begin{array}{l}\text { Overall survival [Median } \\
(95 \% \mathrm{Cl}) \text { (months)] }\end{array}$ & $13.8(9.2,18.4)$ & $8.2(5.7,10.7)$ & $6.3(1.3,11.3)$ & $\begin{array}{l}P<0.0001^{* *} \text { (All wild-type vs. } \\
\text { Any other mutations) }\end{array}$ \\
\hline \multicolumn{5}{|l|}{ Relative dose intensity } \\
\hline Irinotecan [Median (range) (\%)] & $72.8(13.0-100)$ & $81.0(38.4-100)$ & $98.0(49.3-100)$ & $P=0.108^{* * *}$ \\
\hline Cetuximab [Median (range) (\%)] & $86.0(35.7-100)$ & $86.3(11.1-100)$ & $100(80.0-100)$ & $P=0.042^{* * *}$ \\
\hline $\begin{array}{l}\text { Number of treatment cycles } \\
\text { [Median (range)] }\end{array}$ & $12(1-86)$ & $5(1-23)$ & $3(1-12)$ & $P<0.0001^{* * *}$ \\
\hline
\end{tabular}

\footnotetext{
*: Fisher's exact test.
}

${ }^{* *}$ : log rank test.

${ }^{* * *}$ : Kruskal-Wallis test. 


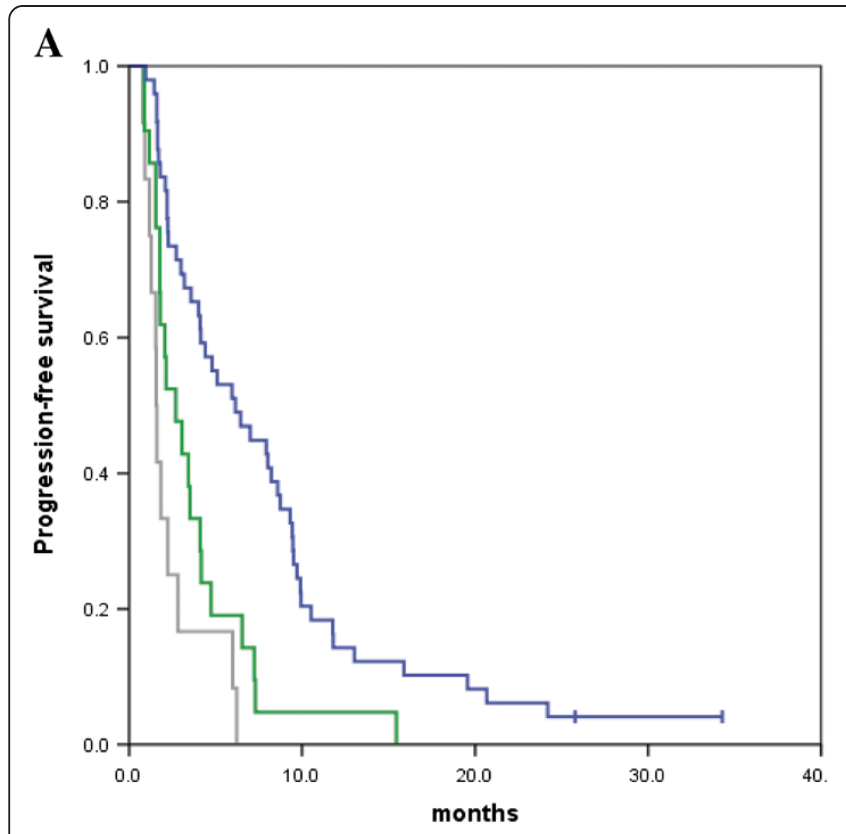

\section{B}

Figure 2 Kaplan-Meier plots of progression-free survival (PFS) and overall survival (OS) according to KRAS, BRAF, NRAS, and PIK3CA gene status. Figure 2A. PFS: Median PFS values were 6.1 months [95\% confidence interval (Cl): 3.1-9.2], 2.7 months (1.2-4.2), and 1.6 months (1.5-1.7) among patients with all wild-type tumors ( $N=49$, blue line), KRAS codon 12 or 13 mutant tumors ( $N=21$, green line), and KRAS codon 61, KRAS codon 146, BRAF, NRAS, or PIK3CA mutant tumors ( $N=12$, gray-line), respectively. Differences in PFS values between patients with all wild-type tumors and those with KRAS codon 61, KRAS codon 146, BRAF, NRAS, or PIK3CA mutant tumors were statistically significant (hazard ratio, 0.22; 95\% Cl, 0.11-0.44; $P<0.0001$ ). Figure 2B. OS: Median OS values were 13.8 months [95\% confidence interval (Cl): 9.2-18.4], 8.2 months (5.7-10.7), and 6.3 months (1.3-11.3) among patients with all wild-type tumors $(N=49$, blue line), with KRAS codon 12 or 13 mutant tumors ( $N=21$, green line), and with KRAS codon 61, KRAS codon 146, BRAF, NRAS, or PIK3CA mutations $(N=12$, gray-line), respectively. Differences in OS values between patients with all wild-type tumors and those with KRAS codon 61, KRAS codon 146, BRAF, NRAS, or PIK3CA mutant tumors were statistically significant (hazard ratio, $0.30 ; 95 \% \mathrm{Cl}, 0.15-0.61 ; P<0.0001$ ).

codon 146, BRAF, NRAS, or PIK3CA mutations (PFS: $P=0.091$, OS: $P=0.236$ ) (Figure $2 \mathrm{~A}$ and $2 \mathrm{~B}$ ).

We also analyzed the differences in PFS and OS between patients with KRAS codon 12 mutations and those with KRAS codon 13 mutations. Similar to our previous study in a different population [17], there were no statistically significant differences between these groups (median PFS: KRAS codon 12, 2.1 months vs. KRAS codon 13, 3.4 months, $\mathrm{P}=0.682$; median OS: KRAS codon 12 , 6.8 months vs. KRAS codon 13, 9.6 months, $\mathrm{P}=0.147$ ).

\section{Discussion}

This study is the first to verify the relevance of the mutation status of KRAS codons 61 and 146, BRAF, NRAS, and PIK3CAto the clinical efficacy of anti-EGFR antibody therapy among Asian patients. As reported in a pooled analysis from a European population, patients with the aforementioned less-frequent mutations exhibited statistically significant worse outcomes equivalent to those of KRAS codon 12 and 13 mutants [8]. Though systemically analyzed studies have not been reported since the first European analysis, our results strongly support the usefulness of the expanded pretreatment test for anti-EGFR therapies.
Because our aim was to compare the outcomes of KRAS codon 12 and 13 mutant cases with those characterized by other mutations, clinical data and FFPE specimens of the patients treated with cetuximab-containing regimens at seven Japanese cancer centers from July 2008 to April 2010 were collected. At that time, the Japanese authorities did not require pretreatment KRAS tests, and patients with KRAS codon 12 and 13 mutations were eventually treated with cetuximab. However, the proportion of patients with KRAS codon 12 or 13 mutant tumors in this study (25.6\%) was slightly lower than that in previous reports of Western and Asian study populations [18], supposedly because several participating institutions had established lab-based tests and used the data for selecting nonbeneficiary populations. Among KRAS codon 12 and 13 wild-type cases, the proportion with mutations of overall tested genes (12/61, $19.7 \%$ ) was similar to that of previous reports, suggesting that such expanded testing would be equally useful in Western and Asian countries.

Because the potential usefulness of multiplex mutation analyses is demonstrated, the development of robust in vitro diagnostic systems is needed for clinical application. The application of multiplex mutation detection systems in colorectal cancer specimens has been reported. 
Lurkin I. et al. reported the validity of multiplex assays using a $\mathrm{SNaPshot}{ }^{\circ}$ Multiplex kit (Life Technologies), which detects 22 mutations in KRAS, BRAF, NRAS, and PIK3CA [19]. Here we evaluated a quality-controlled kit detecting 36 mutations of KRAS codons 61 and 146, BRAF, NRAS, and PIK3CA using Luminex (xMAP) technology. Data obtained by this kit were fully concordant with those by conventional direct sequencing, regardless of any variation in fixation methods between participating institutes (unpublished data).

This kit has several advantages with regard to its development for routine clinical use. It is manufactured under the same quality as the hitherto approved in vitro diagnostic kit detecting mutations in KRAS codons 12 and 13. Design of the hands-on operations is simple and easy; detection of the 36 mutations is performed in a single reaction of multiplex PCR followed by Luminex bead assay, with an overall hands-on time of $4.5 \mathrm{~h}$. In addition, the requirement for template DNA is as low as 50 ng. We collected a median of 370 ng (range: 154889) DNA per $10-\mu \mathrm{m}$ biopsy slice in this study, which is sufficiently large to perform the test and to reserve backup DNA. Meanwhile, the ARMS-Scorpion assay, another approved in vitro diagnostic kit, requires larger amounts of template DNA. The currently approved KRAS codons 12 and 13 kit consists of 8 ( 1 control and 7 mutations) PCR reactions. A total of 80-160 ng of template DNA (10-20 ng for each PCR reaction) are needed to examine a sample [20], and it would be difficult to expand the PCR reactions because of the limitation of template DNA.

It has been estimated that approximately $10 \%-20 \%$ of all patients with colorectal cancer have either KRAS codon 61, KRAS codon 146, BRAF, NRAS, or PIK3CA gene mutations, suggesting that approximately $60,000-$ 120,000 patients $(10 \%-20 \%$ of the 600,000 who die annually from colorectal cancer) worldwide could be screened by this expanded mutation test. Furthermore, because the usefulness of regular administration of aspirin for patients with mutated PIK3CA colorectal cancer and the possibility of combining EGFR and BRAF inhibitors for patients with mutated BRAF colorectal cancer have been reported, detection of those mutations could become of greater importance in many ways $[21,22]$. Once further studies with larger sample sizes and a range of clinical samples provide evidence of its clinical utility, this technique might advance the precision of colorectal cancer treatment.

\section{Conclusions}

Our newly developed multiplex kit is practical and feasible for investigating various types of FFPE samples. Moreover, mutations in KRAS codon 61, KRAS codon 146, BRAF, NRAS, or PIK3CA detected in Asian patients were not predictive of clinical benefits from cetuximab treatment, similar to the result obtained in European studies.

\section{Abbreviations}

EGFR: Anti-epidermal growth factor receptor; PFS: Progression-free survival; OS: Overall survival; Cl: Confidence interval; FFPE: Formalin-fixed, paraffinembedded; CT: Computed tomography; H-E: Hematoxylin-eosin; PCR: Polymerase chain reaction; RR: Response rate; DCR: Disease control rate.

\section{Competing interests}

The authors declare that they have no competing interests.

\section{Authors' contributions}

TY and KT conceived the study design. HB carried out the majority of molecular genetic studies and analyses of the clinical data. ES, TN, KY, KY, SY, and SK provided clinical data and helped collect tumor tissues. SF carried out the pathological diagnoses. TY statistically analyzed the clinical data. AO coordinated the study and helped to draft the manuscript. All authors have read and approved the final manuscript.

\section{Funding}

This study was supported by a Grant-in-Aid for Cancer Research (21 S4-5) from the Ministry of Health, Labour and Welfare of Japan.

\section{Research group members}

Hideaki Bando, Takayuki Yoshino, Katsuya Tsuchihara, Satoshi Fujii, Kohei Shitara, Takeharu Yamanaka, and Atsushi Ohtsu (National Cancer Center Hospital East); Satoshi Yuki and Takahide Sasaki (Hokkaido University); Eiji Shinozaki (Cancer Institute Hospital of Japanese Foundation for Cancer Research); Tomohiro Nishina (Shikoku Cancer Center); Kensei Yamaguchi, Shigenori Kadowaki, and Masako Asayama (Saitama Cancer Center); Kentaro Yamazaki (Shizuoka Cancer Center) and Shinya Kajiura (University of Toyama).

\section{Author details}

${ }^{1}$ Department of Gastroenterology and Gastrointestinal Oncology, National Cancer Center Hospital East, 6-5-1 Kashiwanoha, Kashiwa, Chiba 277-8577, Japan. ${ }^{2}$ Cancer Institute Hospital of Japanese Foundation for Cancer Research, Tokyo, Japan. ${ }^{3}$ National Hospital Organization Shikoku Cancer Center, Ehime, Japan. ${ }^{4}$ Division of Gastrointestinal Oncology, Shizuoka Cancer Center, Shizuoka, Japan. ${ }^{5}$ Division of Gastroenterology, Saitama Cancer Center, Saitama, Japan. ${ }^{6}$ Department of Gastroenterology, Hokkaido University Graduate School of Medicine, Hokkaido, Japan. ${ }^{7}$ The Third Department of Internal Medicine, University of Toyama, Toyama, Japan. ${ }^{8}$ Pathology Division, Research Center for Innovative Oncology, National Cancer Center Hospital East, Chiba, Japan. ${ }^{9}$ Exploratory Oncology Research \& Clinical Trial Center, National Cancer Center, Chiba, Japan.

Received: 30 April 2013 Accepted: 30 August 2013

Published: 3 September 2013

\section{References}

1. Amado RG, Wolf M, Peeters M, Van Cutsem E, Siena S, Freeman DJ, Juan T, Sikorski $R$, Suggs $S$, Radinsky $R$, et al: Wild-type KRAS is required for panitumumab efficacy in patients with metastatic colorectal cancer. J Clin Oncol 2008, 26(10):1626-1634.

2. Karapetis CS, Khambata-Ford S, Jonker DJ, O'Callaghan CJ, Tu D, Tebbutt NC, Simes RJ, Chalchal H, Shapiro JD, Robitaille S, et al: K-ras mutations and benefit from cetuximab in advanced colorectal cancer. N Engl J Med 2008, 359(17):1757-1765.

3. Van Cutsem E, Kohne CH, Hitre E, Zaluski J, Chang Chien CR, Makhson A, D'Haens G, Pinter T, Lim R, Bodoky G, et al: Cetuximab and chemotherapy as initial treatment for metastatic colorectal cancer. N Engl J Med 2009, 360(14):1408-1417.

4. Douillard JY, Siena S, Cassidy J, Tabernero J, Burkes R, Barugel M, Humblet Y, Bodoky G, Cunningham D, Jassem J, et al: Randomized, phase III trial of panitumumab with infusional fluorouracil, leucovorin, and oxaliplatin (FOLFOX4) versus FOLFOX4 alone as first-line treatment in patients with previously untreated metastatic colorectal cancer: the PRIME study. J Clin Oncol 2010, 28(31):4697-4705.

5. Peeters M, Price TJ, Cervantes A, Sobrero AF, Ducreux M, Hotko Y, Andre T, Chan E, Lordick F, Punt CJ, et al: Randomized phase III study of 
panitumumab with fluorouracil, leucovorin, and irinotecan (FOLFIRI) compared with FOLFIRI alone as second-line treatment in patients with metastatic colorectal cancer. J Clin Oncol 2010, 28(31):4706-4713.

6. Vaughn CP, Zobell SD, Furtado LV, Baker CL, Samowitz WS: Frequency of KRAS, BRAF, and NRAS mutations in colorectal cancer. Genes Chromosomes Cancer 2011, 50(5):307-312.

7. Sobrero AF, Maurel J, Fehrenbacher L, Scheithauer W, Abubakr YA, Lutz MP, Vega-Villegas ME, Eng C, Steinhauer EU, Prausova J, et al: EPIC: phase III trial of cetuximab plus irinotecan after fluoropyrimidine and oxaliplatin failure in patients with metastatic colorectal cancer. J Clin Oncol 2008 26(14):2311-2319.

8. De Roock W, Claes B, Bernasconi D, De Schutter J, Biesmans B, Fountzilas G, Kalogeras KT, Kotoula V, Papamichael D, Laurent-Puig P, et al: Effects of KRAS, BRAF, NRAS, and PIK3CA mutations on the efficacy of cetuximab plus chemotherapy in chemotherapy-refractory metastatic colorectal cancer: a retrospective consortium analysis. Lancet Oncol 2010, 11(8):753-762

9. Loupakis F, Ruzzo A, Cremolini C, Vincenzi B, Salvatore L, Santini D, Masi G, Stasi I, Canestrari E, Rulli E, et al: KRAS codon 61, 146 and BRAF mutations predict resistance to cetuximab plus irinotecan in KRAS codon 12 and 13 wild-type metastatic colorectal cancer. Br J Cancer 2009, 101(4):715-721.

10. Di Nicolantonio F, Martini M, Molinari F, Sartore-Bianchi A, Arena S, Saletti P, De Dosso S, Mazzucchelli L, Frattini M, Siena S, et al: Wild-type BRAF is required for response to panitumumab or cetuximab in metastatic colorectal cancer. J Clin Oncol 2008, 26:5705-5712.

11. Perrone F, Lampis A, Orsenigo M, Di Bartolomeo M, Gevorgyan A, Losa M, Frattini M, Riva C, Andreola S, Bajetta E, et al: PI3KCA/PTEN deregulation contributes to impaired responses to cetuximab in metastatic colorectal cancer patients. Ann Oncol 2009, 20(1):84-90.

12. Prenen $H$, De Schutter J, Jacobs B, De Roock W, Biesmans B, Claes B, Lambrechts D, Van Cutsem E, Tejpar S: PIK3CA mutations are not a major determinant of resistance to the epidermal growth factor receptor inhibitor cetuximab in metastatic colorectal cancer. Clin Cancer Res 2009, 15(9):3184-3188

13. Sartore-Bianchi A, Martini M, Molinari F, Veronese S, Nichelatti M, Artale S, Di Nicolantonio F, Saletti P, De Dosso S, Mazzucchelli L, et al: PIK3CA mutations in colorectal cancer are associated with clinical resistance to EGFR-targeted monoclonal antibodies. Cancer Res 2009, 69(5):1851-1857.

14. Itoh Y, Mizuki N, Shimada T, Azuma F, Itakura M, Kashiwase K, Kikkawa E, Kulski JK, Satake M, Inoko H: High-throughput DNA typing of HLA-A, -B, -C, and -DRB1 loci by a PCR-SSOP-Luminex method in the Japanese population. Immunogenetics 2005, 57(10):717-729.

15. Ando A, Shigenari A, Ota M, Sada M, Kawata H, Azuma F, Kojima-Shibata C, Nakajoh M, Suzuki K, Uenishi H, et al: SLA-DRB1 and -DQB1 genotyping by the PCR-SSOP-Luminex method. Tissue antigens 2011, 78(1):49-55.

16. Fukushima Y, Yanaka S, Murakami K, Abe Y, Koshizaka T, Hara H, Samejima C, Kishi Y, Kaneda M, Yoshino T: [High-throughput screening method of KRAS mutations at codons 12 and 13 in formalin-fixed paraffin-embedded tissue specimens of metastatic colorectal cancer]. Gan To Kagaku Ryoho 2011, 38(11):1825-1835.

17. Bando H, Yoshino T, Yuki S, Shinozaki E, Nishina T, Kadowaki S, Yamazaki K, Kajiura S, Tsuchihara K, Fujii S, et al: Clinical outcome of Japanese metastatic colorectal cancer patients harbouring the KRAS p.G13D Mutation treated with cetuximab + Irinotecan. Jpn J Clin Oncol 2012, 42(12):1146-1151.

18. Bando H, Yoshino T, Tsuchihara K, Ogasawara N, Fuse N, Kojima T, Tahara M, Kojima M, Kaneko K, Doi T, et al: KRAS mutations detected by the amplification refractory mutation system-Scorpion assays strongly correlate with therapeutic effect of cetuximab. Br J Cancer 2011, 105(3):403-406

19. Lurkin I, Stoehr R, Hurst CD, van Tilborg AA, Knowles MA, Hartmann A, Zwarthoff EC: Two multiplex assays that simultaneously identify 22 possible mutation sites in the KRAS, BRAF, NRAS and PIK3CA genes. PLoS One 2010, 5(1):e8802.

20. Ogasawara N, Bando H, Kawamoto Y, Yoshino T, Tsuchihara K, Ohtsu A, Esumi H: Feasibility and robustness of amplification refractory mutation system (ARMS)-based KRAS testing using clinically available formalinfixed, paraffin-embedded samples of colorectal cancers. Jpn J Clin Oncol 2011, 41(1):52-56.
21. Prahallad A, Sun C, Huang S, Di Nicolantonio F, Salazar R, Zecchin D, Beijersbergen RL, Bardelli A, Bernards R: Unresponsiveness of colon cancer to BRAF(V600E) inhibition through feedback activation of EGFR. Nature 2012, 483(7387):100-103.

22. Liao X, Lochhead P, Nishihara R, Morikawa T, Kuchiba A, Yamauchi M, Imamura Y, Qian ZR, Baba Y, Shima K, et al: Aspirin use, tumor PIK3CA mutation, and colorectal-cancer survival. N Engl J Med 2012, 367(17):1596-1606.

doi:10.1186/1471-2407-13-405

Cite this article as: Bando et al: Simultaneous identification of 36 mutations in KRAS codons 61 and 146, BRAF, NRAS, and PIK3CA in a single reaction by multiplex assay kit. BMC Cancer 2013 13:405.

\section{Submit your next manuscript to BioMed Central and take full advantage of:}

- Convenient online submission

- Thorough peer review

- No space constraints or color figure charges

- Immediate publication on acceptance

- Inclusion in PubMed, CAS, Scopus and Google Scholar

- Research which is freely available for redistribution

Submit your manuscript at www.biomedcentral.com/submit
C) BioMed Central 